Case Report

\title{
UNILATERAL BICONDYLAR HOFFA FRACTURE WITH A FRACTURE SHAFT AND DISLOCATED KNEE AND HIP IN A MIDDLE AGED ADULT : A RARE CASE REPORT
}

\author{
Ashwin Shetty ${ }^{1}$, Sanath Kumar Shetty ${ }^{2}$, Arjun Ballal ${ }^{3}$, Nirmal Babu ${ }^{4} \&$ Ravindranath Rai ${ }^{5}$ \\ ${ }^{1}$ Associate Professor, ${ }^{2}$ Assistant Professor, ${ }^{3}$ Postgraduate, ${ }^{4}$ Senior Resident, ${ }^{5}$ Professor \& HOD \\ Department Orthopaedic Surgery, K.S. Hegde Medical Academy, Nitte University, \\ M angalore - 575 018, India. \\ Correspondence : \\ Arjun Ballal \\ Postgraduate, Department Orthopaedic Surgery, K.S. Hegde M edical Academy, Nitte University \\ Mangalore - 575018 , India. \\ M obile : +91 $8431644077 \quad$ E-mail : arjchess_Ip@ rediffmail.com
}

\begin{abstract}
: femur, is rare in adults and even rarer in children. comminuted patella and a shaft of femur fracture with a posteriorly dislocated hip.

\section{Introduction:}

Fracture at the distal end of the femur usually occurs in the sagittal plane. ${ }^{1}$ Coronal shear fracture of the distal femoral condyle is an unusual injury. Albert Hoffa first described thisfracture in $1904 .^{2}$
\end{abstract}

Fracture at the distal end of the femur usually occurs in the sagittal plane. Hoffa fracture, i.e., coronal slice fracture of the condyles of the

In the case we wish to discuss here presented to our emergency room with an open knee injury due to a road traffic accident. Imaging of the right lower limb revealed an open Gustilo-Anderson type IIIB bicondylar Hoffa fracture with an anterior dislocation of knee with a

He underwent a successful stabilization and cannulated cancellous screw fixation of the Hoffa fracture and fixation of other injuries.

Keywords: Hoffa fracture, posterior dislocation of hip, Bicondylar, cannulated cancellous screws, Supracondylar fracture of femur

To our best knowledge not many unilateral bicondylar Hoffa fractures have been reported till date.

As this kind of an injury with associated polytrauma to the same limb is a very rare occurance, we would like to discuss it in our case report.

\section{Case Report:}

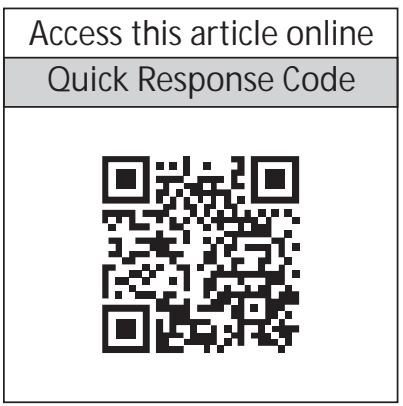

open wound over the right knee with the distal femur protruding from the wound site, with comminuted patella fracture with complete rupture of the cruciate ligaments and the patellar retinaculum of the knee was noted (Figure 1). Contamination was noted at the wound site. His affected hip was noted in flexion, adduction and internal rotation. The limb saturation was noted to be $100 \%$ with no distal neurovascular deficits.

He had no history of any medical complaints and had stable vitals.

Radiography revealed:

- An anterior dislocated right knee with medial and lateral condyle fracture of the right femur (Bicondylar Hoffa fracture), comminuted fracture right patella (Figure 2A)

- Fracture right femur at midshaft (Figure 2B)

- Posteriorly dislocated right hip (Figure 2C)

The limb was splinted and he was started on intravenous fluids and antibiotics and was immediately rushed to the operating room. 


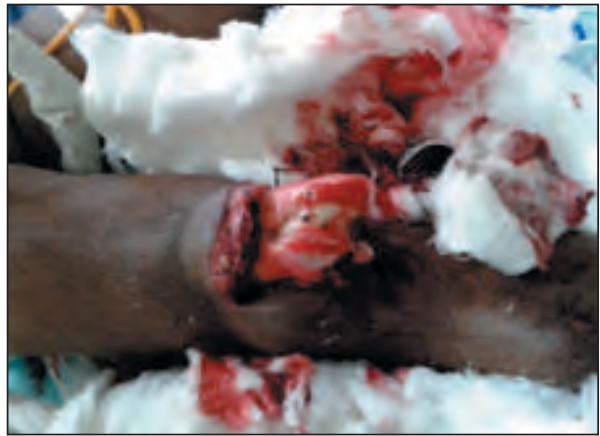

Figure 1: Open wound over the right knee exposing the bicondylar Hoffa fracture and the distal femur and comminuted fracture of patella.

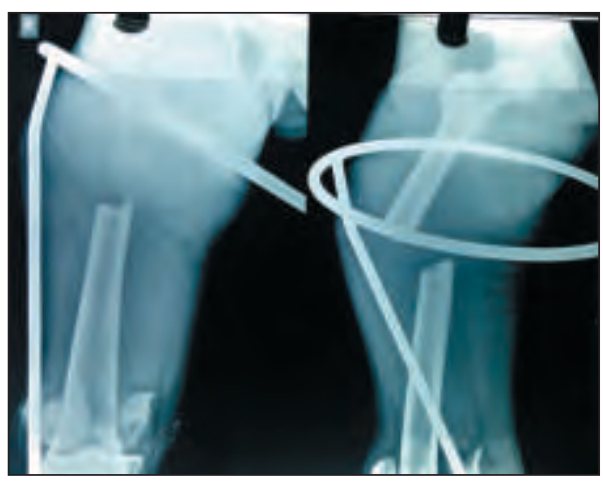

Figure 2B: Fracture right femur at midshaft

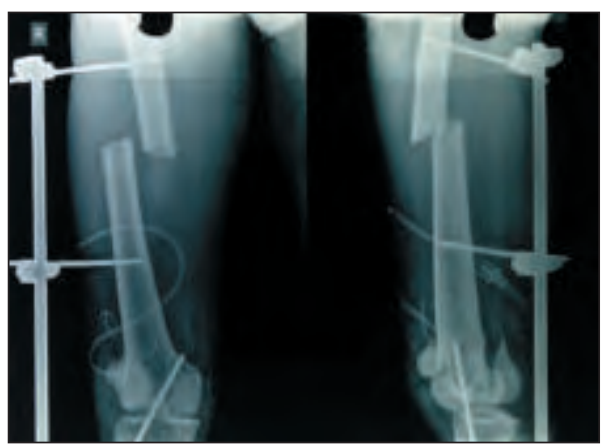

Figure 3: After external fixator application. Note the Steinmann pin Entering from the lateral tibial condyle and exiting through the medial femoral condyle holding the dislocated knee in place.

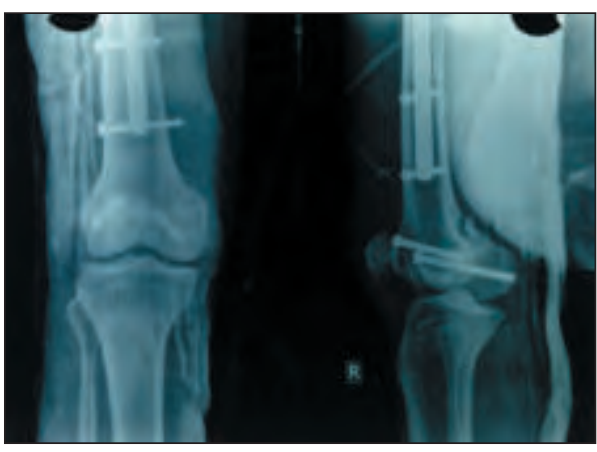

Figure 5: Post-operative $x$-ray right knee showing reduced bicondylar Hoffa fracture with cannulated cancellousscrewsinsitu.

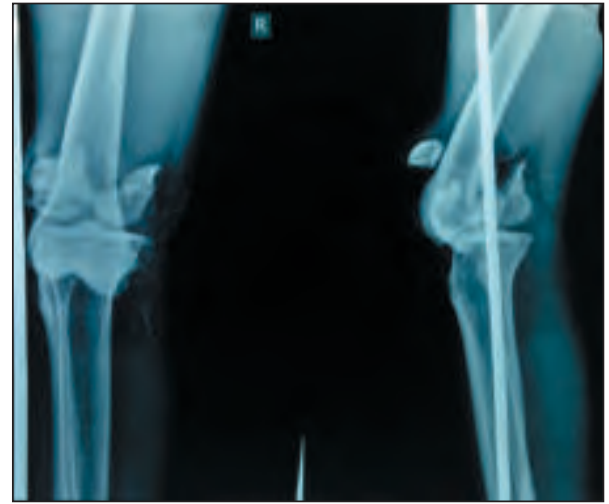

Figure 2A : An anterior dislocated right knee with medial and lateral condyle fracture of the right femur (Bicondylar Hoffa fracture), comminuted fracture right patella.

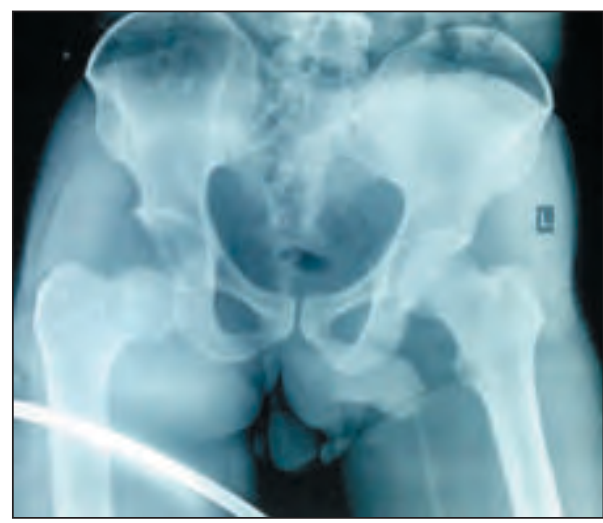

Figure 2C: Posteriorly dislocated right hip.

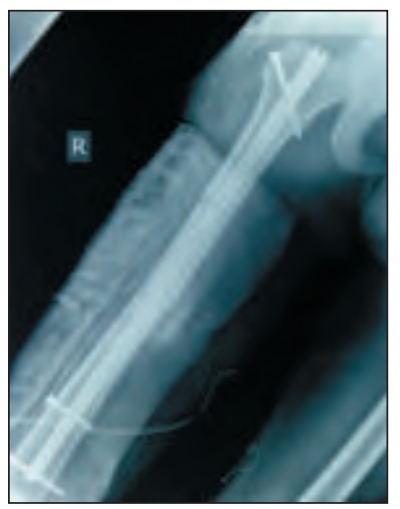

Figure 4 : Post operative x-ray of right femur showing A short femur nail insitu.
Figure 6: On post-operative day 21 the skin looks healthy with no signs of infection. 
In view of the polytrauma and the contamination a staged protocol was planned.

Initially he underwent a thorough debridement of the wound site. The knee was reduced and stabilized using a Steinmann pin from lateral tibial condyle to the medial femoral condyle. A knee spanning external fixator was applied to stabilize the femur shaft fracture and the fracture of the condyles. Then a closed reduction of the hip and partial patellectomy was done (Figure 3).

Patient was maintained on epidural analgesics. Continuous monitoring of vitals and limb saturation was done. Intravenous fluids and antibiotics were continued.

Three days later when the skin conditions improved and no discharge was noted from the wound site, the patient was taken up for a definite fixation.

He underwent: femur nailing with a $11 \times 36$ short femoral nail (Figure 4). Open reduction and internal fixation of the Hoffa fracture was done with $6.5 \mathrm{~mm}$ cannulated cancellous screw fixation (Figure5).

Wound inspection was done on a regular basis to look for any signs of infection. He was continued on intravenous antibiotics till post-operative day 10 . Suture removal was delayed until post operative day 14. On 3 weeks after the surgery the patient was reviewed and examined, the operated site was noted to be clean with no evident signs of infection and skin looked healthy (Figure 6).

\section{Discussion :}

Hoffa fractures are rare injuries, and lateral condyle fractures are more common than medial condyle fractures, probably because of physiological genu valgum; the lateral condyle is the most frequently injured condyle ${ }^{1}$.

\section{References:}

1. Hitesh Lal, Pankaj Bansal, Rahul Khare, and Deepak Mittal, 'Conjoint bicondylar Hoffa fracture in a child': a rare variant treated by minimally invasive approach'. J Orthop Traumatol. Jun 2011; 12(2): 111-114.

2. Hoffa A. Lehrbuch der Frakturen und Luxationen. Stuttgart: Verlag von Ferdinand Enke; 1904. p. 451.

3. Calmet J, Mellado JM, Garci'a Forcada IL, Gine'J (2004) Open bicondylar Hoffa fracture associated with extensor mechanism injury. J Orthop Trauma 18(5):323-325.
Hoffa fracture can be associated with extensor mechanism injuries. ${ }^{3}$. It usually occurs in adults and rarely affects children. The fracture results from a combination of forces: direct trauma, possibly with an element of abduction. Fall on a flexed knee concentrates the force in the posterior half of the femoral condyles, which is why this injury is more common in two-wheeled vehicle accidents where the knee is in a flexed and abducted position ${ }^{1}$.

Letenneur et al. ${ }^{4}$ provided a classification for Hoffa fractures. Type I is a vertical fracture involving the entire condyle parallel to the posterior cortex of the femur. Type II is a fracture of variable size, horizontal to the base of the condyle. They reported the best result with internal fixation and the poorest results in type III.

It is generally accepted that surgical stabilization is necessary to achieve satisfactory function following Hoffa fracture, the reason being that reduction of the fracture fragment is difficult to achieve and maintain by closed reduction and casting/traction techniques, due to the absence of soft tissue attachment ${ }^{1}$.

Operative treatment provides early functional rehabilitation and also decreases the chances of osteoarthritis ${ }^{1}$.

In the literature, there are reports of arthroscopically assisted reduction and internal fixation of Hoffa fracture ${ }^{5}$. Arthroscopic management reduces soft tissue dissection, blood loss, and operative time ${ }^{1}$.

Rehan UI Haq et al reported of a conjoint bicondylar Hoffa fracture in a 17 year old adult ${ }^{6}$.

Hitesh Lal et al reported a case of unilateral bicondylar Hoffa fracture in a 9 year old child ${ }^{1}$.

4. Letenneur J, Labour PE, Rogez JM , Lignon J, Bainvel JV (1978) Fractures de Hoffa: a propos de 20 observations. Ann Chir (Paris) 32(3-4): 213-219.

5. Wallenbock E, Ledinski C (1993) Indications and limits of arthroscopic management of intraarticular fractures of the knee joint. Aktuelle Traumatol 23(2):97-101.

6. Rehan UI Haq, Prashant M odi, IK Dhammi, Anil KJain, Puneet M ishra. 2013. Conjoint bicondylar Hoffa fracture in an adult. Indian journal of Orthopaedics, 47(3): 302-306. 